\section{Brain, Behavior and Evolution}

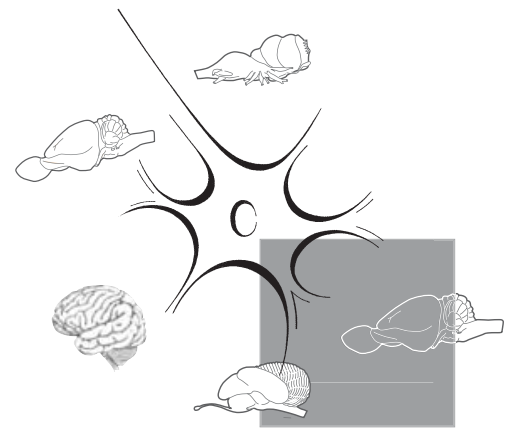

Almost all mammals have distinct tactile hairs that they can use to explore their environment. In particular, laboratory rodents possess about 60 thick, densely innervated facial vibrissae that they sweep in a characteristically rhythmic "whisking" motion with which they gauge the location, shape, texture, and orientation of objects [Fox, 2008]. This mechanosensory information is transmitted to the central nervous system via the trigeminal pathway (Fig. 1a). Much of our current understanding of mammalian tactile senses has been gleaned from the body of literature on the rodent whisker system. Given that research efforts focusing on mice and rats are typically justified by the assumption that these animals are representative of all mammals, including humans, I adopted a comparative neuroanatomical approach in order to test how broadly applicable our knowledge of the rodent trigeminal system is to the trigeminal system of other mammals.

In laboratory rodents, the punctate array of facial vibrissae is represented in a one-to-one fashion in anatomically distinct units in 3 stations of the ascending somatosensory pathway: the brainstem, thalamus, and neocortex. These discrete circular modules are termed barrelettes, barreloids, and barrels, respectively [Woolsey and Van der Loos, 1970; Van der Loos, 1976, Belford and Killackey, 1979; Ma, 1991] (Fig. 1b). Furthermore, within the
Brain Behav Evol 2017;89:65-67

Published online: March 28, 2017

\title{
Conserved Patterns of Trigeminal Somatosensory System Organization in Mammals
}

\author{
Eva K. Sawyer \\ Neuroscience Graduate Program, Vanderbilt University, Nashville, TN, and Department of \\ Bioengineering, University of California, Berkeley, Berkeley, CA, USA
}

brainstem, there are 3 separate and complete somatotopic barrelette representations, the principal sensory nucleus, the spinal trigeminal subnucleus interpolaris, and the spinal trigeminal subnucleus caudalis. Broadly, the principal trigeminal sensory nucleus is vital to the sense of discriminative touch, whereas the subnucleus caudalis is important for pain sensation, and the subnucleus interpolaris may be related to discounting the role of movement during active touch behavior [Diamond et al., 2008].

Clear somatotopic maps make it easy for researchers to identify the disruption of normal somatosensory function, which is one reason why the rodent trigeminal system continues to be used in developmental studies, particularly in investigations of the neocortex. Previous researchers investigating comparative aspects of the trigeminal somatosensory areas have noted that the presence or absence of cortical barrels across species does not correlate well with whisking behavior or phylogeny [Woolsey et al., 1975; Horton and Adams, 2005].

In my own work, I used anatomical and electrophysiological methods to delineate vibrissal representations in the brainstem trigeminal pathway. I suspected that the brainstem relays would have fewer higherlevel integrative functions than the neocortical stations, and would therefore exhibit less interspecific variation than the neocor- tex. I hoped that this reduced variation would make it easier to interpret any differences I did find, at least compared to the seemingly haphazard phylogenetic distribution of neocortical barrels.

One species I selected was the California sea lion (Zalophus californianus), a large amphibious carnivore with long, thick facial vibrissae. Instead of actively whisking, seals and sea lions hold their vibrissae erect and allow hydrodynamic currents to flow by [Miersch et al., 2011]. The whiskers then transduce this motion, allowing the animals to sense minute irregularities in the water flow, such as those generated by a passing fish. My colleagues and I discovered distinct barrelette-like structures in the brainstem of sea lions. Indeed, like rodents, they have 3 distinct sets of brainstem barrelettes [Sawyer et al., 2016] (Fig. 1d).

A second species I investigated was the star-nosed mole (Condylura cristata). This small, fossorial mammal has a distinct tactile "star" consisting of 22 rays that superficially resemble glabrous facial whiskers or fingers (Fig. 1b). Previous works identified

The author won the "Thomas Karger Award for Excellence in Evolutionary Neuroscience". The award was presented at the 2016 J.B. Johnston Club meeting on November 11, 2016.

\section{KARGER}

(C) 2017 S. Karger AG, Base
Eva K. Sawyer

Yartsev Lab, Li Ka Shing Center, Room 275C University of California, Berkeley 1951 Oxford Street, Berkeley, CA 94720-3370 (USA) E-Mail eksawyer@berkeley.edu 


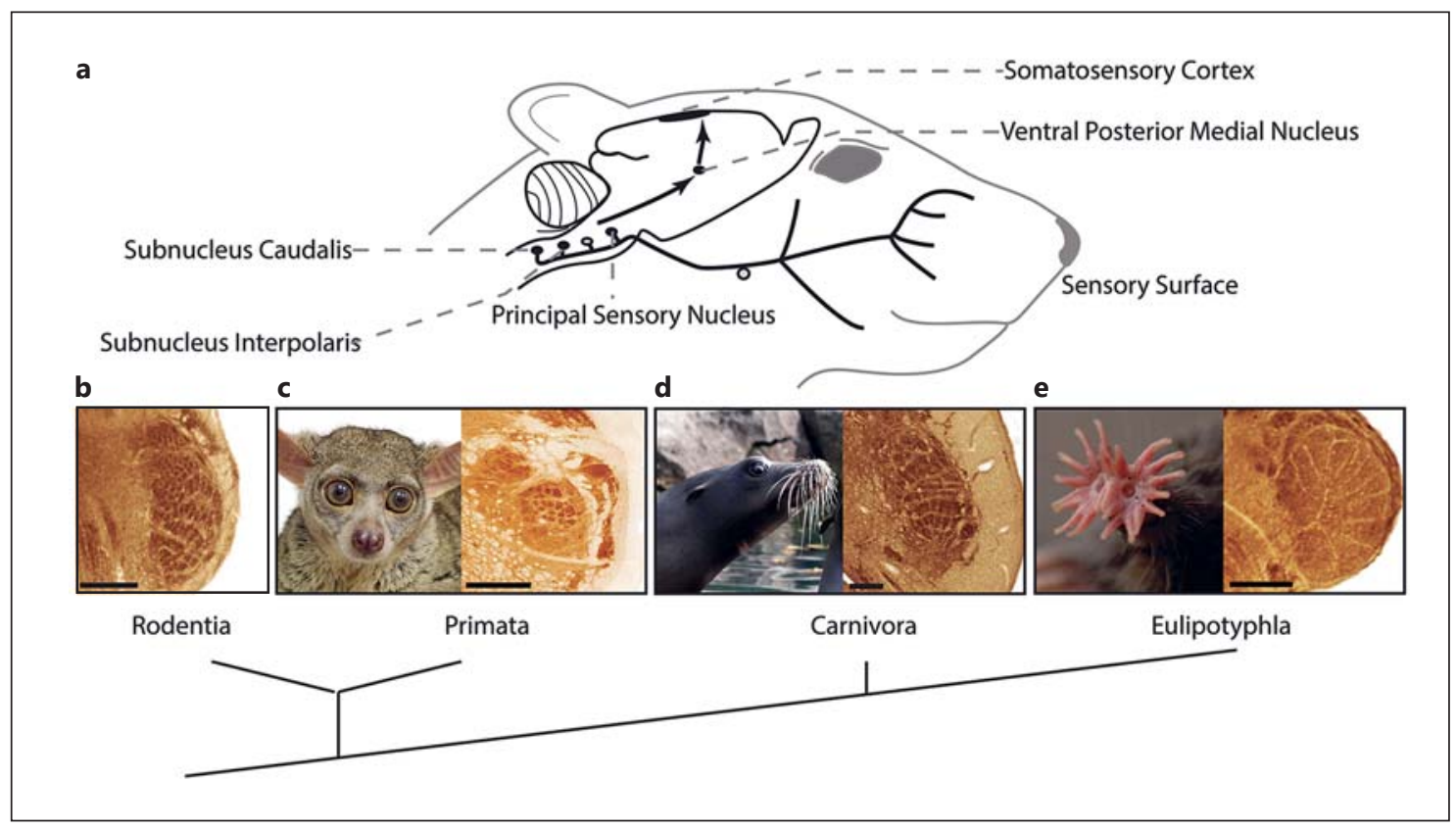

Fig. 1. Conserved patterns of trigeminal organization in mammals. a A schematic of the trigeminal somatosensory pathway in mammals. b A coronal section from the brainstem of the rat (Rattus norvegicus, postnatal day 7) through the spinal trigeminal subnucleus interpolaris $(\mathrm{SpVi})$ stained for cytochrome oxidase (CO). The collection of dark-brown circles reflects the pattern of whisker follicles on the rat's snout. c Left: a prosimian galago (Otolemur garnetti). Right: a coronal section of the galago brainstem (postnatal day 7) through the SpVi and stained for CO; circular modules are evident. d Left: a California sea lion (Zalophus californianus).
Right: a coronal section of the sea lion brainstem (of an approx. 3-month-premature infant) through the SpVi stained for CO; dark circular modules are present and likely represent the facial whisker follicles. e Left: the tactile rays on the nose of a star-nosed mole (Condylura cristata). Right: a coronal section of the star-nosed mole brainstem (on approx. postnatal day 8) through the SpVi stained for CO; the section displays a "star" pattern with 11 stripes that match the 11 rays on each side of the mole's nose. Scale bar, $0.5 \mathrm{~mm}$ for all sections. a distinct star-related pattern in the primary somatosensory cortex and in the principal sensory nucleus of the brainstem [Catania and Kaas, 1995; Catania et al., 2011]. Through in vivo electrophysiological recordings and histochemistry, we uncovered 2 additional star maps in the brainstem, similar to the triplicate rodent "barrelette" system [Sawyer et al., 2014] (Fig. 1e). Importantly, the trigeminal system of star-nosed moles involves glabrous skin (the rays) rather than tactile hairs (whiskers) and is therefore more representative of the human trigeminal system.

Finally, I investigated trigeminal organization in a prosimian primate, the galago or bushbaby (Otolemur garnetti) [Sawyer et al., 2015]. The thin tactile hairs on this basal primate's snout have no significant motor capability and seem unlikely to be highly valued by the animal. Unlike rodents, pinnipeds, or star-nosed moles, the galago is no "champion" of trigeminal somatosen- sation. Instead, galagos are much like humans in terms of their trigeminal somatosensory periphery. Nonetheless, my collaborators and I discovered neural structures in the brainstem (Fig. 1c) related to the galago's tactile hairs. Thus, a key organizational principle of the trigeminal system in tactile specialists is also found in nonspecialists.

Overall, I found visible somatotopic maps in the trigeminal system of all 3 of my chosen species, similar to the maps known from laboratory rodents. Given the broad diversity of these species' habitats, the differences in how they use their trigeminal somatosensory system, and the fact that they belong to 3 different mammalian orders, my findings indicate that the general organization of the trigeminal brainstem nuclei is remarkably conserved.

Nonetheless, some features varied across species. For example, in rats, the vibrissal map in the principal trigeminal nu- cleus is relatively small, while the map in the subnucleus interpolaris is large. In contrast, in star-nosed moles, these 2 maps are similar in size [Ashwell et al., 2006; Catania et al., 2011; Sawyer et al., 2014]. More quantitative comparisons of somatotopic maps, combined with more detailed research into the facial tactile exploratory behavior of these animals, may help to generate novel hypotheses about the specialized sensory functions of these brainstem nuclei. Furthermore, these comparisons could be extended to the somatosensory thalamic nuclei and the cortex. Lastly, it seems possible that modular representation of functionally important facial areas (e.g., the lips and adjacent skin) exist in human somatosensory areas, especially in the brainstem, even though they have not yet been observed. 


\section{References}

Ashwell KW, Hardman CD, Paxinos G (2006): Cyto- and chemoarchitecture of the sensory trigeminal nuclei of the echidna, platypus and rat. J Chem Neuroanat 31:81-107.

Belford GR, Killackey HP (1979): The development of vibrissae representation in subcortical trigeminal centers of the neonatal rat. J Comp Neurol 188:63-74.

Catania KC, Kaas JH (1995): Organization of the somatosensory cortex of the star-nosed mole. J Comp Neurol 351:549-567.

Catania KC, Leitch DB, Gauthier D (2011): A star in the brainstem reveals the first step of cortical magnification. PLoS One 6:e22406.

Diamond ME, von Heimendahl M, Knutsen PM, Kleinfeld D, Ahissar E (2008): 'Where' and 'what' in the whisker sensorimotor system. Nat Rev Neurosci 9:601-612.

Fox K (2008): Barrel Cortex. New York, Cambridge University Press.
Horton JC, Adams DL (2005): The cortical column: a structure without a function. Philos Trans R Soc Lond B Biol Sci 360:837862.

Ma PM (1991): The barrelettes - architectonic vibrissal representations in the brainstem trigeminal complex of the mouse. I. Normal structural organization. J Comp Neurol 309: 161-199.

Miersch L, Hanke W, Wieskotten S, Hanke FD, Oeffner J, Leder A, Brede M, Witte M, Dehnhardt G (2011): Flow sensing by pinniped whiskers. Philos Trans R Soc Lond B Biol Sci 366:3077-3084.

Sawyer EK, Leitch DB, Catania KC (2014): Organization of the spinal trigeminal nucleus in star-nosed moles. J Comp Neurol 522:33353350.

Sawyer EK, Liao CC, Qi HX, Balaram P, Matrov D, Kaas JH (2015): Subcortical barrelette-like and barreloid-like structures in the prosimian galago (Otolemur garnetti). Proc Natl Acad Sci USA 112:7079-7084.

Sawyer EK, Turner EC, Kaas JH (2016): Somatosensory brainstem, thalamus, and cortex of the California sea lion (Zalophus californianus). J Comp Neurol 524:1957-1975.

Van der Loos H (1976): Barreloids in mouse somatosensory thalamus. Neurosci Lett 2:1-6.

Woolsey TA, Van der Loos H (1970): The structural organization of layer IV in the somatosensory region (SI) of mouse cerebral cortex. The description of a cortical field composed of discrete cytoarchitectonic units. Brain Res 17:205-242.

Woolsey TA, Welker C, Schwartz RH (1975): Comparative anatomical studies of the $\mathrm{SmL}$ face cortex with special reference to the occurrence of "barrels" in layer IV. J Comp Neurol 164:79-94. 